\title{
Absence of an independent association between serum uric acid and left ventricular mass in Caucasian hypertensive women and men
}

\author{
G. Mulè*, E. Nardi, M. Costanzo, M. Mogavero, L. Guarino, T. Viola, \\ M.G. Vario, V. Cacciatore, G. Andronico, G. Cerasola, S. Cottone
}

Dipartimento di Medicina Interna e Specialistica (DIMIS), Cattedra di Medicina Interna e Cattedra di Nefrologia, European Society of Hypertension Excellence Centre, Università di Palermo, Via del Vespro, 129, 90127 Palermo, Italy

Received 4 September 2011; received in revised form 9 December 2011; accepted 18 January 2012

\author{
KEYWORDS \\ Essential \\ hypertension; \\ Serum uric acid; \\ Cardiovascular risk; \\ Left ventricular mass; \\ Left ventricular \\ hypertrophy
}

\begin{abstract}
Background and aim: Experimentally uric acid may induce cardiomyocyte growth and interstitial fibrosis of the heart. However, clinical studies exploring the relationship between serum uric acid (SUA) and left ventricular (LV) mass yielded conflicting results.

The aim of our study was to evaluate the relationships between SUA and LV mass in a large group of Caucasian essential hypertensive subjects.

Methods and results: We enrolled 534 hypertensive patients free of cardiovascular complications and without severe renal insufficiency. In all subjects routine blood chemistry, including SUA determination, echocardiographic examination and $24 \mathrm{~h}$ ambulatory blood pressure (BP) monitoring were obtained.

In the overall population we observed no significant correlation of SUA with LV mass indexed for height ${ }^{2.7}\left(\mathrm{LVMH}^{2.7}\right)(r=0.074)$. When the same relationship was analysed separately in men and women, we found a statistically significant correlation in female gender $(r=0.27$; $p<0.001)$, but not in males $(r=-0.042 ; p=N S)$. When we grouped the study population in sex-specific tertiles of SUA, an increase in $\mathrm{LVMH}^{2.7}$ was observed in the highest tertiles in women $\left(44.5 \pm 15.6\right.$ vs $47.5 \pm 16$ vs $\left.55.9 \pm 22.2 \mathrm{~g} / \mathrm{m}^{2.7} ; p<0.001\right)$, but not in men.

The association between SUA and $\mathrm{LVMH}^{2.7}$ in women lost statistical significance in multiple regression analyses, after adjustment for age, $24 \mathrm{~h}$ systolic BP, body mass index, serum creatinine and other potential confounders.

Conclusions: Our findings do not support an independent association between SUA and LV mass in Caucasian men and women with arterial hypertension.

(c) 2012 Elsevier B.V. All rights reserved.
\end{abstract}

\footnotetext{
* Corresponding author. Via Monte San Calogero, 29, 90146 Palermo, Italy. Tel.: +39 91 6554578; fax: +39 916554331.

E-mail address: giuseppe.mule@unipa.it (G. Mulè).
} 


\section{Introduction}

Hyperuricemia is particularly common in hypertensive patients, especially in those with metabolic syndrome (MetS) [1-4], where may be a marker of hyperinsulinemia [5] and insulin resistance [5], as well as of renal dysfunction $[1,3,6,7]$ and diuretic use $[1,3]$. In some, but not in all, studies $[1,2,8]$ performed in hypertensive subjects, the association between serum uric acid (SUA) and cardiovascular (CV) events remained significant after adjustment for potential confounding factors.

The development of left ventricular hypertrophy (LVH), in hypertensive patients with high concentration of SUA may potentially explain, at least in part, the enhanced CV risk observed in these subjects, because LVH is a strong predictor of CV events [9]. Indeed, uric acid is thought to induce experimentally cardiomyocyte growth and interstitial fibrosis of the heart, in part via activation of the renin-angiotensin system (RAS) $[10,11]$ and in part by inducing endothelin-1 gene expression in cardiac fibroblasts [12].

However, clinical studies exploring the relationship between SUA and left ventricular (LV) mass yielded conflicting results [8,13-22]. Moreover, in some studies a significant effect of SUA on LV mass (LVM) was observed only in women $[14,16]$, while others described the opposite [15].

The aim of our study was to evaluate in a large group of Caucasian essential hypertensive subjects the relationship between SUA and LVM and to assess the influence of gender, if any, on this relationship.

\section{Methods}

The population of this cross-sectional study was selected from 646 hypertensive Caucasian patients consecutively attending our Hypertension Unit.

The exclusion criteria were: secondary or malignant hypertension, heart failure, positive history or clinical signs of ischemic heart disease, cerebrovascular disease, severe renal function impairment, defined by an estimated glomerular filtration rate (GFR) $<30 \mathrm{ml} / \mathrm{min} / 1.73 \mathrm{~m}^{2}$, gout, treatment with SUA-lowering medication (with the exception of losartan) in the last year, dyslipidemia requiring pharmacological treatment, type 1 diabetes or type 2 diabetes requiring insulin therapy, major non cardiovascular diseases.

Fifty-two subjects met these criteria and therefore were not enrolled.

Of the remaining individuals 60 were also excluded, because suboptimal echocardiographic tracings or fewer than $80 \%$ valid ambulatory blood pressure monitoring (ABPM) readings were obtained. Hence, the final statistical analysis involved 534 patients.

Before entering the study, 311 hypertensive subjects had been pharmacologically treated. These patients were studied at least 2 weeks after the discontinuation of all antihypertensive drugs.

All the participants to this study may be considered as sedentary subjects.

Persons who reported smoking cigarettes regularly during the past year were considered current smokers.
Informed consent was obtained from each patient. The study protocol was approved by the local review board.

\section{Study design}

After the period of pharmacological washout, clinic blood pressure (BP) was recorded by a doctor, in the nondominant arm using the auscultatory method with a mercury sphygmomanometer and appropriated cuff for arm diameter, following the 2007 guidelines of the European Society of Hypertension (ESH) [23]. Three consecutive measurements were taken at 2 min intervals and averaged.

The following morning, after an overnight fast of at least $12 \mathrm{~h}$, blood samples were drawn to perform routine blood chemistry.

Furthermore, $24 \mathrm{~h} \mathrm{ABPM}$ and echocardiographic study were carried out.

\section{Measurements}

Determination of routine biochemical parameters was performed with standard techniques by using an autoanalyser (ILab 300+, Instrumentation Laboratory, Milan, Italy).

Serum uric acid was measured using an uricase/peroxidase method implemented in the above mentioned autoanalyser.

Estimated GFR was calculated using the Chronic Kidney Disease Epidemiology Collaboration (CKD-EPI) equation [24].

Low-density lipoprotein cholesterol (LDL) was calculated by the Friedwald formula.

The presence of type 2 diabetes was defined as fasting plasma glucose of $126 \mathrm{mg} / \mathrm{dl}$ or higher or use of oral hypoglycemic agents.

The diagnosis of the MetS was based on the joint interim statement of the International Diabetes Federation Task Force on Epidemiology and Prevention; National Heart, Lung, and Blood Institute; American Heart Association; World Heart Federation; International Atherosclerosis Society; and International Association for the Study of Obesity [25].

A portable, non-invasive Spacelabs 90207 recorder (Redmond, Washington, USA) performed the $24 \mathrm{~h}$ ABPM.

$M$-mode measurements were taken with the American Society of Echocardiography (ASE) recommendations [26]. LVM was determined using the ASE corrected cube-formula [27]. It was indexed by both body surface area (LVMI) and by height elevated by a power of $2.7\left(\mathrm{LVMH}^{2.7}\right)$, in order to provide a more stringent allowance for obesity [28].

A cut-off point of $51 \mathrm{~g} / \mathrm{m}^{2.7}$ in either gender was set to separate normal from LVH [29].

Left ventricular hypertrophy was defined also as LVMI $\geq 125 \mathrm{~g} / \mathrm{m}^{2}$ for men and $\geq 110 \mathrm{~g} / \mathrm{m}^{2}$ for women, as suggested by the 2007 guidelines of the ESH [23].

\section{Statistics}

The study population was separated into two groups of male and female participants. We split also the study population in sex-specific tertiles of SUA (division points for females: 3.8 and $5 \mathrm{mg} / \mathrm{dl}$; for males: 4.9 and $6 \mathrm{mg} / \mathrm{dl}$ ), in 
patients with and without MetS and in subjects with and without LVH.

Normally distributed continuous variables are given as means \pm SD. Categorical variables are presented as percentages.

Normal distribution of the continuous variables was assessed using the Kolmogorov-Smirnov test and the assumption of satisfactory Gaussian distribution was met for all of the examined variables, except for duration of hypertension, triglycerides, LVM, LVMI, LVMH ${ }^{2.7}$ and SUA which showed a positive skewed distribution. Because the application of non parametric tests to LVM, LVMI, $\mathrm{LVMH}^{2.7}$ and SUA or their mathematical transformations provided similar results to those obtained using parametric tests or untransformed data, we have used only the latter in this paper. Conversely, duration of hypertension and triglycerides, for their very high level of skewness, were expressed as median and interquartile range and logarithmically transformed before starting the statistical tests.

Differences between subgroups were evaluated using one-way analysis of variance (ANOVA) and the independentsample Student's $t$-test for continuous variables and the chi-square test for the categorical variables.

Two-way ANOVA was used to assess the differences regarding SUA in subjects with and without MetS on the basis of gender.

Tukey post-hoc test was used to perform multiple comparisons.

Adjustment for age and other potential confounders was carried out by analysis of covariance (ANCOVA).

Univariate relations between SUA and LVM and of these parameters with other variables in the overall population and in either gender were tested by Pearson correlation coefficients and linear regression analysis.

Linear multiple stepwise regression analyses were used to explore the independent correlates of $\mathrm{LVMH}^{2.7}$ and of LVMI in the overall population and in both sexes. Three models were built. The first (model 1 ) included age, sex (only in the whole population), $24 \mathrm{~h}$ systolic and diastolic
BPs, known duration of hypertension, previous pharmacological treatment and SUA. Model 2 included SUA, the covariates that had shown a significant association with LVM in model 1 plus triglycerides, high density lipoprotein (HDL) cholesterol and body mass index (BMI). Model 3 included SUA, the covariates that had shown a significant association with LVM in model 2 plus serum creatinine. Subsequently in these models $24 \mathrm{~h}$ BPs, BMI and serum creatinine were replaced respectively by clinic BPs, waist circumference and estimated GFR. Finally, all these models were run again after the exclusion of the 40 diabetic participants.

The null hypothesis was rejected at a two-tailed $p<0.05$.

The statistical analyses were performed using the SYSTAT DATA software package, version 12 (Systat, San Jose, CA, USA).

\section{Results}

Table 1 summarises demographic and clinical data of the overall population, subdivided according to gender.

The overall prevalence of MetS was $46.4 \%$. It was higher in females $(51.3 \%)$ than in males $(42.7 \% ; p=0.05)$. SUA values were higher in subjects with MetS than in those without it, in the whole population $(5.4 \pm 1.5$ vs $4.8 \pm 1.4 \mathrm{mg} / \mathrm{dl} ; p<0.001)$, in men $(5.9 \pm 1.3 \mathrm{vs}$ $5.2 \pm 1.4 \mathrm{mg} / \mathrm{dl} ; p<0.001)$, and in women $(4.9 \pm 1.3 \mathrm{vs}$ $4.1 \pm 1.2 \mathrm{mg} / \mathrm{dl} ; p<0.001)$.

SUA levels did not differ in diabetic $(4.8 \pm 1.5 \mathrm{mg} / \mathrm{dl})$ compared to non diabetic subjects $(5.1 \pm 1.5 \mathrm{mg} / \mathrm{dl}$; $p=0.33)$, in smokers $(5.2 \pm 1.6 \mathrm{mg} / \mathrm{dl})$ versus non smokers $(5.0 \pm 1.4 \mathrm{mg} / \mathrm{dl} ; \quad p=0.33)$, in never treated $(5.1 \pm 1.4 \mathrm{mg} / \mathrm{dl})$ versus previously pharmacologically treated patients $(5.0 \pm 1.5 \mathrm{mg} / \mathrm{dl} ; p=0.13)$. SUA concentrations were also similar in subjects previously treated with losartan $(n=24 ; 5.0 \pm 1.6 \mathrm{mg} / \mathrm{dl})$ versus the remaining ones $(5.1 \pm 1.4 \mathrm{mg} / \mathrm{dl} ; p=0.73)$, as well as in patients previously treated with thiazides $(n=97)$ or loop

Table 1 Demographics and clinical data of the overall study population and of the two groups divided according to gender.

\begin{tabular}{|c|c|c|c|c|}
\hline & Overall study population & Males & Females & $p$ \\
\hline & $(n=534)$ & $(n=300)$ & $(n=234)$ & \\
\hline Age, years & $47.1 \pm 13.7$ & $45.1 \pm 14$ & $49.9 \pm 12.9$ & $<0.001$ \\
\hline Known hypertension duration, months ${ }^{a}$ & $6(2-13)$ & $5(1-12)$ & $6(2-14)$ & 0.091 \\
\hline Previous antihypertensive treatment, \% & 58.2 & 50.7 & 67.9 & $<0.001$ \\
\hline Serum uric acid, $\mathrm{mg} / \mathrm{dl}$ & $5.07 \pm 1.5$ & $5.5 \pm 1.4$ & $4.5 \pm 1.3$ & $<0.001$ \\
\hline Body mass index, $\mathrm{Kg} / \mathrm{m}^{2}$ & $28.4 \pm 4.8$ & $28.4 \pm 4.2$ & $28.5 \pm 5.5$ & 0.812 \\
\hline Waist circumference, $\mathrm{cm}$ & $96.2 \pm 14$ & $99.5 \pm 11.7$ & $92 \pm 15.5$ & $<0.001$ \\
\hline Glycemia, mg/dl & $98.3 \pm 24.6$ & $97.3 \pm 22.4$ & $94.6 \pm 27.4$ & 0.211 \\
\hline Total cholesterol, mg/dl & $204.7 \pm 39.4$ & $202.6 \pm 39$ & $207.4 \pm 39.9$ & 0.163 \\
\hline HDL cholesterol, mg/dl & $46.1 \pm 10.7$ & $44.7 \pm 9.9$ & $48.3 \pm 11.3$ & $<0.001$ \\
\hline LDL cholesterol, mg/dl & $131.7 \pm 36.2$ & $130.2 \pm 36.5$ & $133.8 \pm 35.7$ & 0.254 \\
\hline Triglycerides, mg/dl ${ }^{\mathrm{a}}$ & $118(80-165)$ & $128(83-175.5)$ & $108.5(76-149)$ & 0.003 \\
\hline Prevalence of diabetes, $\%$ & 7.5 & 6.7 & 8.5 & 0.514 \\
\hline Serum creatinine, mg/dl & $0.88 \pm 0.18$ & $0.96 \pm 0.2$ & $0.78 \pm 0.16$ & $<0.001$ \\
\hline Estimated GFR, $\mathrm{ml} / \mathrm{min} / 1.73 \mathrm{~m}^{2}$ & $78.5 \pm 20.7$ & $70.5 \pm 16.6$ & $88.9 \pm 20.9$ & $<0.001$ \\
\hline Current smokers, $\%$ & 27.2 & 32.0 & 21.0 & 0.004 \\
\hline
\end{tabular}

${ }^{a}$ Data of these variables, for their skewed distribution, were given as medians and interquartile range. 
diuretics $(n=10)$, always in combination with other antihypertensive drugs, when compared to the remaining subjects (5.2 \pm 1.4 vs $5.0 \pm 1.4 \mathrm{mg} / \mathrm{dl} ; p=0.19)$.

Clinic and $24 \mathrm{~h}$ BP values and main echocardiographic parameters of the study population, analysed also on the basis of gender, are reported in Table 2 .

Table 3 shows some demographic and clinical characteristics of the study population separated in sex-specific tertiles of SUA. Prevalence of LVH and values of LVMI and of $\mathrm{LVMH}^{2.7}$ increased in highest SUA tertiles only in women.

Univariate analysis of correlation showed (see Supplementary table S1) that among the whole group, SUA was directly related to age, BMI, WC, sCreat, triglycerides, IVS, PWT, LVIDD, RWT, LAD, and inversely related to HDL cholesterol, and estimated GFR. In both sexes SUA was correlated to BMI, WC, sCreat, estimated GFR, whereas only in men it was positively related to total and LDL cholesterol and inversely associated with HDL cholesterol. Only in women SUA was correlated to age, IVS, PWT, RWT, and $L A D$ and $E / A$ ratio. No significant correlation was found between SUA and clinic or $24 \mathrm{~h}$ BPs in the overall study population and in men and women separately analysed.

The Supplementary table S2 displays the univariate correlations of LVMH ${ }^{2.7}$ and of LVMI with other variables.

In the whole study population, SUA did not significantly correlate neither with $\operatorname{LVMH}^{2.7}(r=0.074, p=0.09$; Fig. 1), nor with LVMI $(r=0.075 ; p=0.08)$. This was true even when the relationships between these variables were assessed by a non parametric test (Spearman's correlation analysis), instead of the Pearson's coefficient ( $\rho=0.081$, $p=0.06 ; \rho=0.083, p=0.055$, respectively).

Similarly, SUA was not associated neither to $\mathrm{LVMH}^{2.7}$ $(r=-0.04, p=0.55)$, nor to LVMI $(r=-0.03, p=0.60)$ in the subset $(n=223)$ of patients never pharmacologically treated with antihypertensive agents.

Taking each gender into consideration separately, we found a significant correlation between SUA and LVMH ${ }^{2.7}$ in women, but not in men (Fig. 1). Likewise, LVMI was significantly associated to SUA in females $(r=0.20$, $p<0.001)$, but not in males $(r=-0.07, p=0.10)$.

No difference was found regarding LVMH $^{2.7}(49.8 \pm 18.8$ vs $47.7 \pm 16.8 \mathrm{mg} / \mathrm{dl} ; p=0.26)$ or RWT (39.4 \pm 8.8 vs $38.7 \pm 7.5 \% ; p=0.41$ ) in patients previously treated with diuretics when compared to the remaining subjects.

In all the study subjects, those with $\mathrm{LVH}$, defined as a value of $\mathrm{LVMH}^{2.7}>51 \mathrm{~g} / \mathrm{m}^{2.7}$, showed slightly, but not significantly, higher levels of SUA $(5.3 \pm 1.7$ vs $5.0 \pm 1.7$; $p=0.07)$. Whereas in the group of male subjects the concentration of SUA in the two subgroups with and without LVH was virtually the same $(5.5 \pm 1.7$ vs $5.5 \pm 1.5$; $p=0.97)$, in the group of women those with LVH had greater values of SUA than women without LVH $(4.9 \pm 1.3$ vs $4.3 \pm 1.3 ; p<0.001)$, but this difference became not significant $(p=0.75)$ after adjustment for age, sCreat, $24 \mathrm{~h}$ systolic BP, BMI, triglycerides and previous antihypertensive therapy.

Analogous results were obtained when LVH was defined as $L V M I \geq 110 \mathrm{~g} / \mathrm{m}^{2}$ in women and $>125 \mathrm{~g} / \mathrm{m}^{2}$ in men (data not shown).

Table 2 Clinic and $24 \mathrm{~h}$ blood pressures and echocardiographic parameters of the overall study population and of the two groups divided according to gender.

\begin{tabular}{|c|c|c|c|c|}
\hline & $\begin{array}{l}\text { Overall study } \\
\text { population } \\
(n=534)\end{array}$ & $\begin{array}{l}\text { Males } \\
(n=300)\end{array}$ & $\begin{array}{l}\text { Females } \\
(n=234)\end{array}$ & $p$ \\
\hline Clinic systolic blood pressure, $\mathrm{mmHg}$ & $159.1 \pm 21.5$ & $157.1 \pm 20.4$ & $161.0 \pm 22.6$ & 0.037 \\
\hline Clinic diastolic blood pressure, $\mathrm{mmHg}$ & $94.6 \pm 13.7$ & $96.0 \pm 13.8$ & $93.1 \pm 13.4$ & 0.015 \\
\hline $24 \mathrm{~h}$ systolic blood pressure, $\mathrm{mmHg}$ & $137.6 \pm 12.7$ & $136.0 \pm 12.7$ & $139.1 \pm 12.6$ & 0.005 \\
\hline $24 \mathrm{~h}$ diastolic blood pressure, $\mathrm{mmHg}$ & $85.7 \pm 10.7$ & $87.2 \pm 10.4$ & $84.1 \pm 10.9$ & $<0.001$ \\
\hline End-diastolic left ventricular internal dimension (LVIDD), mm & $50.3 \pm 6.5$ & $51.9 \pm 6.2$ & $48.1 \pm 6.3$ & $<0.001$ \\
\hline End-diastolic interventricular septum thickness (IVS), mm & $10.4 \pm 1.9$ & $10.7 \pm 1.9$ & $9.9 \pm 1.9$ & $<0.001$ \\
\hline End-diastolic posterior wall thickness (PWT), mm & $9.7 \pm 1.8$ & $10 \pm 1.7$ & $9.3 \pm 1.8$ & $<0.001$ \\
\hline Myocardial relative wall thickness (RWT), \% & $39.0 \pm 8.2$ & $38.8 \pm 7.6$ & $39.3 \pm 8.9$ & 0.443 \\
\hline Left atrial dimension (LAD), mm & $36.1 \pm 5.6$ & $36.9 \pm 4.8$ & $35.1 \pm 6.3$ & $<0.001$ \\
\hline Ejection fraction (EF), \% & $64.2 \pm 6.6$ & $63.7 \pm 6.0$ & $64.9 \pm 7.2$ & 0.04 \\
\hline Early/late diastolic peak flow velocity (E/A) ratio & $1.20 \pm 0.41$ & $1.26 \pm 0.44$ & $1.11 \pm 0.35$ & $<0.001^{\mathrm{a}}$ \\
\hline Left ventricular mass, $g$ & $189 \pm 68$ & $207.1 \pm 60$ & $165.8 \pm 69$ & $<0.0001$ \\
\hline Left ventricular mass indexed for height ${ }^{2.7}, \mathrm{~g} / \mathrm{m}^{2.7}$ & $48.6 \pm 17.7$ & $47.8 \pm 16.7$ & $49.6 \pm 18.9$ & $0.244^{b}$ \\
\hline Left ventricular mass index, $\mathrm{g} / \mathrm{m}^{2}$ & $102.1 \pm 35$ & $105.7 \pm 34.8$ & $97.3 \pm 34.7$ & $0.006^{b}$ \\
\hline Prevalence of left ventricular hypertrophy (A), \% & 32.0 & 30.0 & 34.6 & 0.298 \\
\hline Prevalence of left ventricular hypertrophy (B), \% & 23.0 & 22.3 & 23.9 & 0.74 \\
\hline
\end{tabular}

(A): Left ventricular hypertrophy was defined as $\mathrm{LVMH}^{2.7}>51 \mathrm{~g} / \mathrm{m}^{2.7}$ in either gender.

(B): Left ventricular hypertrophy was defined as LVMI $\geq 125 \mathrm{~g} / \mathrm{m}^{2}$ for men and $\geq 110 \mathrm{~g} / \mathrm{m}^{2}$ for women.

$\left(p=0.277\right.$ for left ventricular mass indexed for height ${ }^{2.7}$ and $p=0.002$ for left ventricular mass index).

a This difference disappeared after adjustment for age.

b Similar results were obtained by using Kruskal-Wallis test, instead of Student's $t$-test, for the slightly skewed distribution of these variables. 
Table 3 Descriptive characteristics of the patients on the basis of tertiles of serum uric acid and gender.

\begin{tabular}{|c|c|c|c|c|c|c|c|c|}
\hline & \multicolumn{4}{|l|}{ Males } & \multicolumn{4}{|l|}{ Females } \\
\hline & $\begin{array}{l}\text { I Tertile } \\
(n=100)\end{array}$ & $\begin{array}{l}\text { II Tertile } \\
(n=98)\end{array}$ & $\begin{array}{l}\text { III Tertile } \\
(n=102)\end{array}$ & $p$ & $\begin{array}{l}\text { I Tertile } \\
(n=78)\end{array}$ & $\begin{array}{l}\text { II Tertile } \\
(n=77)\end{array}$ & $\begin{array}{l}\text { III tertile } \\
(n=79)\end{array}$ & $p$ \\
\hline Age, years & $44.1 \pm 14.5$ & $43.4 \pm 13.5$ & $47.4 \pm 13.8$ & 0.096 & $45.2 \pm 12.3^{\wedge \wedge}$ & $50.7 \pm 12.8^{\S}$ & $53.3 \pm 12.3$ & $<0.001$ \\
\hline Known hypertension duration, months & $4(1.25-12)$ & $5(1.5-12)$ & $6(1-15)$ & 0.337 & $5(2-11.3)$ & $5(2-12.3)$ & $9.5(3-16.5)$ & 0.092 \\
\hline Previous antihypertensive treatment, \% & 51 & 53.3 & 48.1 & 0.769 & 68.4 & 73 & 63.1 & 0.412 \\
\hline Body mass index, $\mathrm{Kg} / \mathrm{m}^{2}$ & $27.2 \pm 3.5^{\wedge \wedge}$ & $28.1 \pm 3.5^{*}$ & $29.7 \pm 5.1$ & $<0.001$ & $27.1 \pm 4.9^{\wedge}$ & $27.9 \pm 5.6^{*}$ & $30.1 \pm 5.6$ & 0.002 \\
\hline Waist circumference, $\mathrm{cm}$ & $96.5 \pm 8.9^{\wedge \wedge}$ & $98.1 \pm 8.8^{\wedge}$ & $102.5 \pm 10.7$ & $<0.001$ & $87.8 \pm 13.1^{\wedge \wedge}$ & $89.8 \pm 14.5^{* *}$ & $97.1 \pm 16.5$ & $<0.001$ \\
\hline Glycemia, mg/dl & $98.7 \pm 32$ & $96.2 \pm 12.4$ & $97.0 \pm 17.8$ & 0.525 & $96.8 \pm 30.1$ & $102.1 \pm 30.8$ & $99.6 \pm 21.1$ & 0.525 \\
\hline Total cholesterol, mg/dl & $194.3 \pm 39$ & $207.7 \pm 40.2$ & $206.1 \pm 37.1$ & 0.578 & $203.6 \pm 35.0$ & $208.3 \pm 37.5$ & $210.1 \pm 45.8$ & 0.578 \\
\hline HDL cholesterol, mg/dl & $45.8 \pm 10.7$ & $45.1 \pm 9.8$ & $43.2 \pm 9.1$ & 0.191 & $50.3 \pm 10.6$ & $47.3 \pm 11.7$ & $47.5 \pm 11.6$ & 0.267 \\
\hline LDL cholesterol, mg/dl & $123.9 \pm 36$ & $131.9 \pm 36.6$ & $135.0 \pm 36.5$ & 0.107 & $130.8 \pm 31.9$ & $136.3 \pm 38.0$ & $133.9 \pm 36.9$ & 0.066 \\
\hline Triglycerides, mg/dl & $113(75-154)^{*}$ & $142(85-185)^{\S}$ & $134(93-185)$ & 0.01 & $105(79-132)^{*}$ & $103.5(75-149)$ & $115(79-183.8)$ & 0.018 \\
\hline Prevalence of metabolic syndrome, \% & $29.0^{\wedge \wedge}$ & $44.6^{\S}$ & 53.7 & 0.001 & $31.6^{\wedge \wedge}$ & $54.1^{\S \S}$ & 66.7 & $<0.001$ \\
\hline Prevalence of diabetes, $\%$ & 9.0 & 3.2 & 7.4 & 0.261 & 9.2 & 10.8 & 6 & 0.535 \\
\hline Serum creatinine, mg/dl & $0.93 \pm 0.17^{\wedge \wedge}$ & $0.93 \pm 0.14^{*}$ & $1.01 \pm 0.18$ & $<0.001$ & $0.73 \pm 0.14^{\wedge \wedge}$ & $0.76 \pm 0.15^{*}$ & $0.83 \pm 0.18$ & 0.001 \\
\hline Estimated GFR, $\mathrm{ml} / \mathrm{min} / 1.73 \mathrm{~m}^{2}$ & $73.8 \pm 18.9^{\wedge \wedge}$ & $72.8 \pm 15.2^{*}$ & $65.5 \pm 14.1$ & $<0.001$ & $96.3 \pm 19.9^{\wedge \wedge}$ & $89.9 \pm 20.3^{*}$ & $81.5 \pm 19.9$ & $<0.001$ \\
\hline Current smokers, \% & 41.7 & 27.0 & 31.3 & 0.089 & 24 & 22 & 19.1 & 0.653 \\
\hline Clinic systolic blood pressure & $157.2 \pm 19.3$ & $159.9 \pm 22.1$ & $154.3 \pm 19.8$ & 0.154 & $157.0 \pm 19.0$ & $160.9 \pm 19.4$ & $165.1 \pm 27.3$ & 0.072 \\
\hline Clinic diastolic blood pressure & $95.2 \pm 13.6$ & $97.7 \pm 15.9$ & $95.1 \pm 11.9$ & 0.359 & $92.0 \pm 13.1$ & $94.9 \pm 14.1$ & $92.5 \pm 13.1$ & 0.358 \\
\hline $24 \mathrm{~h}$ systolic blood pressure & $136.8 \pm 11.4$ & $136.3 \pm 13.1$ & $135.0 \pm 13.5$ & 0.626 & $138.3 \pm 11.4$ & $139.7 \pm 14.6$ & $139.4 \pm 12.1$ & 0.772 \\
\hline $24 \mathrm{~h}$ diastolic blood pressure & $86.7 \pm 9.8$ & $87.3 \pm 10.7$ & $87.7 \pm 10.8$ & 0.821 & $85.7 \pm 9.9$ & $83.9 \pm 12.0$ & $82.7 \pm 10.7$ & 0.224 \\
\hline LV mass indexed for height ${ }^{2.7}, \mathrm{~g} / \mathrm{m}^{2.7}$ & $50.9 \pm 20.3$ & $45.4 \pm 13$ & $47.1 \pm 15.5$ & 0.07 & $44.5 \pm 15.6$ & $47.5 \pm 16$ & $55.9 \pm 22.2$ & $<0.001$ \\
\hline LV mass index, $\mathrm{g} / \mathrm{m}^{2}$ & $112.0 \pm 42.5$ & $103.4 \pm 29$ & $102.6 \pm 30.3$ & 0.103 & $91.9 \pm 31.8^{*}$ & $93.2 \pm 26.3$ & $105.8 \pm 41.8$ & 0.019 \\
\hline Myocardial relative wall thickness, \% & $37.9 \pm 7.0$ & $39.6 \pm 8.5$ & $38.9 \pm 7.4$ & 0.32 & $38.6 \pm 9.9$ & $38.3 \pm 7.5$ & $41.0 \pm 8.1$ & 0.11 \\
\hline Prevalence of $\operatorname{LVH}(A)^{a}, \%$ & 35.4 & 23 & 31.1 & 0.18 & $21.4^{\wedge \wedge}$ & 32.4 & 48.1 & 0.002 \\
\hline Prevalence of LVH $(B)^{a}$, \% & 29 & 17.3 & 20.6 & 0.275 & $15.8^{* *}$ & $18.9^{*}$ & 35.7 & 0.006 \\
\hline
\end{tabular}

${ }^{*} p<0.05$ vs III Tertile; ${ }^{* *} p<0.01$ vs III Tertile; ${ }^{\wedge} p<0.005$ vs III Tertile; ${ }^{\wedge} p<<0.001$ vs III Tertile; ${ }^{\S} p<0.05$ vs I Tertile; ${ }^{\S \S} p<0.01$ vs I Tertile.

${ }^{a}$ For the definition of left ventricular hypertrophy (LVH) (A) and (B) see legend of Table 2. 


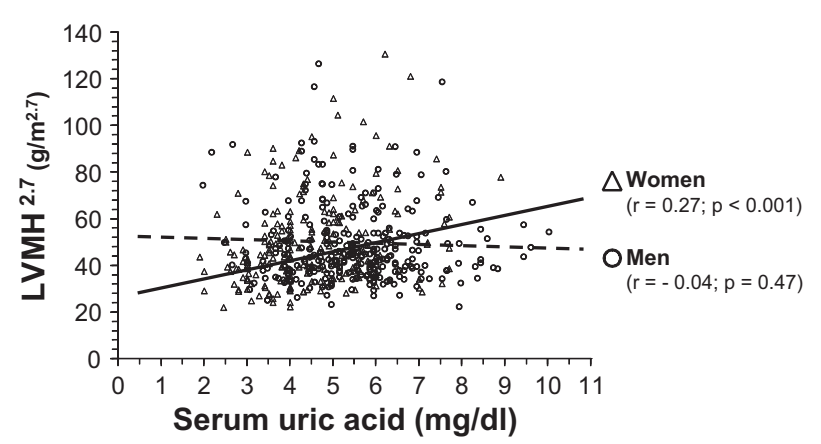

Figure 1 Scattergram showing the relationship between serum uric acid and left ventricular mass indexed for height ${ }^{2.7}$ $\left(\mathrm{LVMH}^{2.7}\right)$ in 534 essential hypertensive patients. The relationship was not statistically significant in the whole study population $(r=0.074 ; p=0.09)$ and in men (open circles) $(r=-0.042 ; p=0.47)$. In women (open triangles) the same relationship was positive and statistically significant $(r=0.271 ; p<0.001)$. The calculated regression lines for males (broken line) and females (solid line) are also shown. The use of Spearman's rank correlation analysis, instead of the Pearson's coefficient, yielded similar results $(\rho=0.081$, $p=0.06$, in the whole population; $\rho=-0.012, p=0.84$, in males; $\rho=0.243, p<0.001$, in females).

Multiple linear regression analyses confirmed that SUA was unrelated to $\mathrm{LVMH}^{2.7}$ in the whole study population $(p=0.78)$, in men $(p=0.13)$ and in women $(p=0.19)$ (Table 3). Similarly, when in the multivariable model we replaced the dependent variable $\mathrm{LVMH}^{2.7}$ with LVMI, this latter was not independently associated with SUA ( $p=0.63$ in the overall study population, $p=0.12$ in men and $p=0.40$ in women).
The independent correlates of $\mathrm{LVMH}^{2.7}$ and of LVMI (age, $24 \mathrm{~h}$ systolic BP, serum creatinine, BMI) were the same in the overall population, in men and in women.

Table 4 shows the results of multiple regression analyses performed to assess the independent predictors of $\mathrm{LVMH}^{2.7}$ in the female group.

The inclusion into the multivariate models of clinic, instead of the $24 \mathrm{~h}$ ambulatory BP readings, of WC instead of BMI and of estimated GFR instead of sCreat did not significantly modify the results. The same was true when diabetic patients were excluded from the analysis.

The univariate associations observed in women between SUA and RWT and between SUA and E/A ratio (Table S1) disappeared after adjustment for age, $24 \mathrm{~h}$ systolic BP, $\mathrm{sCreat}$ and $\mathrm{BMI}$ in multiple regression analyses.

\section{Discussion}

The most relevant result of this study is the absence of an independent association between SUA levels and LVM, regardless of the method of indexation. Even if in univariate analyses, in the women group, SUA was closely related to $\mathrm{LVMH}^{2.7}$ and to $\mathrm{LVH}$, these associations lost statistical significance after adjustment for many potential confounders.

Our findings seem to be in contrast with experimental studies that support a pathogenetic role of hyperuricemia in the development of LVH. Previous reports have shown that uric acid induces endothelial dysfunction and smooth muscle cell proliferation by activating the RAS and inflammatory mediators such as tumor necrosis factor-alpha and mitogen-activated protein kinases, which are known to induce cardiac hypertrophy $[1,10,11,30]$. It has been also reported that uric acid in vitro may increase endothelin-1

Table 4 Multiple regression models to assess the independent correlates of left ventricular mass indexed for height ${ }^{2.7}$ $\left(\mathrm{LVMH}^{2.7}\right)$ in female gender.

\begin{tabular}{|c|c|c|c|c|c|c|}
\hline & \multicolumn{2}{|c|}{ Model 1} & \multicolumn{2}{|c|}{ Model 2} & \multicolumn{2}{|c|}{ Model 3} \\
\hline & \multicolumn{2}{|c|}{ Multiple $R: 0.530$} & \multicolumn{2}{|c|}{ Multiple $R: 0.566$} & \multicolumn{2}{|c|}{ Multiple $R: 0.593$} \\
\hline & \multicolumn{2}{|c|}{$\begin{array}{l}\text { Adjusted multiple } R^{2} \text { : } \\
0.267\end{array}$} & \multicolumn{2}{|c|}{$\begin{array}{l}\text { Adjusted multiple } R^{2} \text { : } \\
0.307\end{array}$} & \multicolumn{2}{|c|}{$\begin{array}{l}\text { Adjusted multiple } R^{2} \text { : } \\
0.339\end{array}$} \\
\hline & $\beta$ & $p$ & $\beta$ & $p$ & $\beta$ & $p$ \\
\hline Age & 0.279 & $<0.001$ & 0.263 & $<0.001$ & 0.243 & $<0.001$ \\
\hline $24 \mathrm{~h}$ systolic blood pressure & 0.369 & $<0.001$ & 0.289 & $<0.001$ & 0.290 & $<0.001$ \\
\hline Serum uric acid & 0.170 & 0.006 & 0.120 & 0.046 & - & NS \\
\hline $\begin{array}{l}\text { Previous antihypertensive } \\
\text { treatment }\end{array}$ & 0.118 & 0.052 & - & NS & & \\
\hline $24 \mathrm{~h}$ diastolic blood pressure & - & NS & & & & \\
\hline $\begin{array}{l}\text { Known hypertension duration } \\
\text { (Log) }\end{array}$ & - & NS & & & & \\
\hline Body mass index & & & 0.228 & $<0.001$ & 0.266 & $<0.001$ \\
\hline HDL cholesterol & & & - & NS & & \\
\hline Triglycerides (Log) & & & - & NS & & \\
\hline Serum creatinine & & & & & 0.216 & $<0.001$ \\
\hline
\end{tabular}

Model 1 includes the following covariates: age, $24 \mathrm{~h}$ systolic and diastolic BPs, duration of hypertension, previous treatment and SUA. Model 2 includes the covariates significantly associated with $\mathrm{LVMH}^{2.7}$ in model 1 plus triglycerides, HDL cholesterol and BMI. Model 2 includes the covariates significantly associated with $\mathrm{LVMH}^{2.7}$ in model 2 plus serum creatinine. $\beta$ : standardised regression coefficient NS: not significant. 
gene expression in rat cardiac fibroblasts [12]. Indeed, a significant positive relation between SUA and LVM or LVH has been reported in some studies conducted in Asian populations [8,13-15]. Kurata et al. reported that SUA levels correlated positively with LVMI in male hypertensive patients, but not in females [15]. In a smaller sample of hypertensive subjects Matsumura et al found the opposite [14]. Iwashima et al., in 619 hypertensive individuals, showed that SUA was independently associated with LVMI [8]. More recently, Mitsuhashi et al. reported that SUA was positively associated with electrocardiographically diagnosed LVH in healthy Japanese men [13].

Moreover, Viazzi et al., in 425 middle-aged hypertensive subjects, in univariate analysis, found a correlation between SUA and LVMI, only in women, but the statistical significance was just on the borderline level( $p=0.04)$ [16].In female gender, the probability of having LVH was higher in subjects with elevated SUA levels in multiple logistic regression analysis $(p=0.03)$, but the multivariate model did not include relevant covariates, known to be associated with SUA or LVM, such as BMI and systolic BP [16]. Finally, in 1225 newly diagnosed hypertensive patients, SUA was related to LVMI in univariate analysis [17]. However, this relationship became barely significant after adjustment for multiple confounders, not including, like in the previous study, BMI and systolic BP [17].

On the other hand, our results are in agreement with several other studies conducted chiefly in hypertensive populations [18-22]. Campo et al. found that hyperuricemia was not an independent correlate of LVH in a study sample of 677 male hypertensives [18]. Tsioufis et al., studied 842 patients with essential hypertension and found that increased SUA levels were associated with microalbuminuria but not with LVH [19]. Similarly, Cuspidi et al., in a cross-sectional study of 580 untreated hypertensive patients, failed to detect an independent association of SUA with LVMI [20]. In another Italian study, including 400 essential hypertensives, SUA did not significantly correlate with $\mathrm{LVMH}^{2.7}$ in multivariate analyses after taking into account estimated GFR and indices of insulin resistance [21]. More recently, in the general population of the Vobarno Study, including 385 subjects from Northern Italy, no independent association was observed between SUA and LVM [22].

The discrepant conclusions of these studies may in part be related to methodological differences and to heterogeneity in patient characteristics.

The conflicting findings about the relationships between SUA and LVM seem in part to mirror the controversy on the role of SUA as independent risk factor for CV morbidity and mortality $[1,2,8]$. Up until today, it is still unclear whether hyperuricemia has a causative role in CVD or, as has been more often suggested, is simply a marker for other risk factors, frequently associated with increased SUA levels, such as hypertension, dyslipidemia, MetS and renal dysfunction [1].

In agreement with the literature, our study confirms that SUA concentrations are closely related to most components of the MetS $[1-6,16,19,20]$, including BMI or WC, triglycerides, HDL cholesterol and with the MetS itself, as well as with indices of renal function such as $\mathrm{sCreat}$ and estimated GFR $[1-3,6,7,15,21]$. The association of SUA with these parameters, most of which are well known correlates of LVM, largely explained the significant univariate relationships that we found in female gender between SUA and cardiac hypertrophy. Indeed, we noticed that these associations became not significant after adjustment for these factors in multivariate analyses.

There are some potential limitations of our study.

The cross-sectional design of the present investigation does not allow us to evaluate the impact of hyperuricemia over time.

Furthermore, previous antihypertensive treatment that had been stopped may have affected the results because of the persisting influence on cardiac mass, even though patients' blood pressure and SUA levels may have returned to control values. However, in the subset of hypertensive patients who had never pharmacologically treated we found results similar to those obtained in the whole population, with regard to LVM. Moreover, the absence of association between SUA and LVM was confirmed, also taking into account previous treatment in multivariate analyses.

Finally, it is important to note that our results were obtained in a selected population of Caucasian middle-aged patients with mild-to-moderate essential hypertension, without severe renal function impairment, free of cardiovascular diseases. Therefore, the conclusions of our study cannot be extrapolated to non white populations and caution is needed when applying the results of our investigation to different clinical settings.

In conclusion, our study showed that serum uric acid is not independently associated with left ventricular mass, regardless of the method of indexation, in both sexes.

On the basis of our findings, if hyperuricemia plays a causative role in the pathogenesis of cardiovascular disease, it is unlikely that this could be in part mediated by the development of left ventricular hypertrophy.

\section{Acknowledgements}

This work was supported in part by the Italian Ministry for University and Scientific Research (MURST) grants ("ex 60\% quotas").

\section{Appendix. Supplementary material}

Supplementary data associated with this article can be found, in the online version, at doi:10.1016/j.numecd. 2012.01.007.

\section{References}

[1] Feig D, Kang DH, Johnson R. Uric acid and cardiovascular risk. N Engl J Med 2008;359:1811-21.

[2] Strazzullo P, Puig JG. Uric acid and oxidative stress: relative impact on cardiovascular risk? Nutr Metab Cardiovasc Dis 2007; 17:409-14. doi:10.1016/j.numecd.2007.02.011.

[3] Lin CS, Hung YJ, Chen GY, Tzeng TF, Lee DY, Chen CY, et al. A multicenter study of the association of serum uric acid, serum creatinine, and diuretic use in hypertensive patients. Int J Cardiol 2011;148:325-30. doi:10.1016/j.ijcard.2009.11.024. 
[4] Onat A, Uyarel H, Hergenc G, Karabulut A, Albayrak S, Sari I, et al. Serum uric acid is a determinant of metabolic syndrome in a population-based study. Am J Hypertens 2006;19:1055-62.

[5] Bonora E, Capaldo B, Cavallo Perin P, Del Prato S, De Mattia G, Frittitta $L$, et al. Hyperinsulinemia and insulin resistance are independently associated with plasma lipids, uric acid and blood pressure in non-diabetic subjects. the GISIR database. Nutr Metab Cardiovasc Dis 2008;18:624-31. doi:10.1016/j. numecd.2007.05.002.

[6] Viazzi F, Leoncini G, Ratto E, Falqui V, Parodi A, Conti N, et al. Mild hyperuricaemia and subclinical renal damage in untreated primary hypertension. Am J Hypertens 2007;20: 1276-82.

[7] Cerasola G, Mulè G, Nardi E, Cusimano P, Palermo A, Arsena R, et al. Clinical correlates of renal dysfunction in hypertensive patients without cardiovascular complications: the REDHY study. J Hum Hypertens 2010;24:44-50.

[8] Iwashima Y, Horio T, Kamide K, Rakugi H, Ogihara T, Kawano Y. Uric acid, left ventricular mass index, and risk of cardiovascular disease in essential hypertension. Hypertension 2006;47: 195-202.

[9] Vakili BA, Okin PM, Devereux RB. Prognostic implications of left ventricular hypertrophy. Am Heart J 2001;141:334-41. doi:10.1067/mhj.2001.113218.

[10] Corry DB, Eslami P, Yamamoto K, Nyby MD, Makino H, Tuck ML. Uric acid stimulates vascular smooth muscle cell proliferation and oxidative stress via the vascular renin-angiotensin system. J Hypertens 2008;26:269-75.

[11] Yu MA, Sanchez-Lozada LG, Johnson RJ, Kang DH. Oxidative stress with an activation of the renin-angiotensin system in human vascular endothelial cells as a novel mechanism of uric acid-induced endothelial dysfunction. J Hypertens 2010;28: 1234-42.

[12] Cheng TH, Lin JW, Chao HH, Chen YL, Chen CH, Chan P, et al. Uric acid activates extracellular signal-regulated kinases and thereafter endothelin-1 expression in rat cardiac fibroblasts. Int J Cardiol 2010;139:42-9. doi:10.1016/j.ijcard.2008.09.004.

[13] Mitsuhashi H, Yatsuya H, Matsushita K, Zhang $H$, Otsuka R, Muramatsu T, et al. Uric acid and left ventricular hypertrophy in Japanese men. Circ J 2009;73:667-72.

[14] Matsumura K, Ohtsubo T, Oniki H, Fujii K, lida M. Genderrelated association of serum uric acid and left ventricular hypertrophy in hypertension. Circ J 2006;70:885-8.

[15] Kurata A, Shigematsu Y, Higaki J. Sex-related differences in relations of uric acid to left ventricular hypertrophy and remodeling in Japanese hypertensive patients. Hypertens Res 2005;28:133-9.

[16] Viazzi F, Parodi D, Leoncini G, Parodi A, Falqui V, Ratto E, et al. Serum uric acid and target organ damage in primary hypertension. Hypertension 2005;45:991-6.

[17] Xaplanteris P, Vlachopoulos C, Vyssoulis G, TerentesPrintzios D, Alexopoulos N, Lazaros G, et al. Uric acid levels, left ventricular mass and geometry in newly diagnosed, never treated hypertension. J Hum Hypertens 2011;25:340-2.

[18] Campo C, Ruilope LM, Segura J, Rodicio JL, Garcia-Robles R, Garcia-Puig J. Hyperuricemia, low urine urate excretion and target organ damage in arterial hypertension. Blood Press 2003;12:277-83.

[19] Tsioufis C, Chatzis D, Vezali E, Dimitriadis K, Antoniadis D, Zervoudaki A, et al. The controversial role of serum uric acid in essential hypertension: relationships with indices of target organ damage. J Hum Hypertens 2005;19:211-7.

[20] Cuspidi C, Valerio C, Sala C, Meani S, Esposito A, Zanchetti A, et al. Lack of association between serum uric acid and organ damage in a never-treated essential hypertensive population at low prevalence of hyperuricemia. Am J Hypertens 2007;20: 678-85.

[21] Perticone F, Maio R, Ruberto C, Cassano S, Tripepi G, Perticone $M$, et al. Kidney function and risk factors for left ventricular hypertrophy in untreated uncomplicated essential hypertension. Am J Kidney Dis 2008;52:74-84. doi:10.1053/j. ajkd.2008.02.302.

[22] Salvetti M, Muiesan ML, Paini A, Agabiti Rosei C, Aggiusti C, Stassaldi $D$, et al. Relationship between serum uric acid and arterial stiffness and cardiac and carotid artery structure in a general population in Northern Italy. J Hypertens; 2010: e588-9.

[23] The Task Force for the Management of Arterial Hypertension of the European Society of Hypertension (ESH) and of the European Society of Cardiology (ESC). Guidelines for the management of arterial hypertension. J Hypertens 2007;25:1105-87.

[24] Levey A, Stevens LA, Schmid CH, Zhang YL, Castro 3rd AF, Feldman $\mathrm{HI}$, et al. A new equation to estimate glomerular filtration rate. Ann Intern Med 2009;150:604-12.

[25] Alberti KG, Eckel RH, Grundy SM, Zimmet PZ, Cleeman JI, Donato KA, et al. Harmonizing the metabolic syndrome: a joint interim statement of the International Diabetes Federation Task Force on Epidemiology and Prevention; National Heart, Lung, and Blood Institute; American Heart Association; World Heart Federation; International Atherosclerosis Society; and International Association for the Study of Obesity. Circulation 2009;120:1640-5.

[26] Sahn DJ, DeMaria A, Kisslo J, Weiman A. The committee on Mmode standardization of the American society of echocardiography. Circulation 1978;58:1072-3.

[27] Lang RM, Bierig M, Devereux RB, Flachskampf FA, Foster E, Pellikka PA, et al. Recommendations for chamber quantification. Eur J Echocardiography 2006;7:79-108.

[28] de Simone G, Daniels SR, Devereux RB, Meyer RA, Roman MJ, de Divitiis $O$, et al. Left ventricular mass and body size in normotensive children and adults: assessment of allometric relations and the impact of overweight. J Am Coll Cardiol 1992;20:1251-60. doi:10.1016/0735-1097(92)90385-Z.

[29] Liao Y, Cooper RS, Durazo-Arvizu R, Mensah GA, Ghali GK. Prediction of mortality risk by different methods of indexation for left ventricular mass. J Am Coll Cardiol 1997;29:641-7. doi:10.1016/S0735-1097(96)00552-9.

[30] Kanellis J, Watanabe S, Li JH, Kang DH, Li P, Nakagawa T, et al. Uric acid stimulates monocyte chemoattractant protein1 production in vascular muscle cells via mitogen-activated protein kinase and cycloxygenase-2. Hypertension 2003;41: 1287-93. 